\title{
LA REFORMA DE LA REFORMA
}

\author{
Rafael Feito Alonso \\ Universidad Complutense
}

\begin{abstract}
RESUMEN. El actual gobierno se ha propuesto acabar con los aspectos más progresistas de la LOGSE. Basta con recordar que el PP fue el único partido parlamentario que votó negativamente esta ley. Había un aspecto que disgustaba de un modo especial a esta formación política: su igualitarismo. Desde el primer momento el PP puso de manifiesto que no le gustaba lo más mínimo la comprensividad para el grupo de edad 14-16 años.

Este artículo se estructurará en cuatro grandes apartados. En primer lugar se hará una valoración de la LOGSE, especialmente de lo que pudo haber sido y no lo fue. En segundo lugar me referiré al debate virtual entre los profesores de secundaria que tuvo lugar en la página web del ministerio de educación. En tercer lugar trataré de analizar cuáles son las intenciones contra-reformistas del actual gobierno. Acabaré, a modo de conclusiones, con una valoración de la situación actual en un momento de neo-conservadurismo rampante.

ABSTRACT. Spanish government in office dislikes the educational law passed by the former socialist goverment. In fact, Popular Party -the party backing the governmentopposed the educational law passed by the Parliament in 1990. Popular Party seemed to specially dislike the egalitarism in the law. Popular Party fought the idea of comprehensiveness till the end of the first level of secondary education -till sixteen years-.

This paper will be structured along four paragraphs. The first one is devoted to an appraisal of the education act of 1991, specially what it come have becomen and have not. The second one has to do with a very interesting debate in internet among teachers. Thridly I will try to analyze the intentions of the government. And finally, I will show a reflection on what is hapenning today wiyth special references to a new educational conservatism.
\end{abstract}

El actual gobierno se ha propuesto acabar con los aspectos más progresistas de la LOGSE. Basta con recordar que el PP fue el único partido parlamentario que votó negativamente esta ley. Había un aspecto que disgustaba de un modo especial a esta formación política: su igualitarismo. Desde el primer momento el PP puso de manifiesto que no le gustaba lo más mínimo la comprensividad para el grupo de edad 14-16 años.

De acuerdo con la actual ministra son tres los objetivos bajo los que se escuda el actual proceso de contra-reforma: elevar la calidad de la docencia y del aprendizaje, preparar adecuadamente a las nuevas generaciones y promover una "auténtica igualdad de oportunidades". El apoyo empírico de este proceso descansa en tres pilares: las jornadas de reflexión sobre la ESO auspiciadas por el MEC en diciembre de 199, el dictamen sobre la enseñanza de las humanidades de la Comisión presidida por Díaz Ambrona y el diagnóstico sobre el rendimientos de los escolares de 14 y de 16 años efectuado por el INCE. 
Hoy en día, desgraciadamente, la LOGSE no solo cuenta con la oposición de la derecha política. Como veremos hay incluso sectores del profesorado que se declaran a sí mismos de izquierdas que se han visto atenazados por las contradicciones del proceso de reforma, atenazados hasta el extremo de subirse al carro del rechazo de la comprensividad. Es esta una cuestión que veremos al analizar el debate virtual sobre la ESO.

Estructuraré esta exposición en cuatro grandes apartados. En primer lugar haré una valoración de la LOGSE, especialmente de lo que pudo haber sido y no lo fue. En segundo lugar me referiré al debate virtual entre los profesores de secundaria que tuvo lugar en la página web del ministerio de educación. En tercer lugar trataré de analizar cuáles son las intenciones contra-reformistas del actual gobierno. Acabaré, a modo de conclusiones, con una valoración de la situación actual en un momento de neo-conservadurismo rampante.

\section{La LOGSE que puedo ser}

Vaya por delante que la situación educativa conformada por la LOGSE es considerablemente mejor que la anterior. Incluso los datos del INCE muestran que los alumnos de la ESO son levemente mejores -y eso que solo hablamos de los componentes cognitivos- que los de los anteriores tramos equivalentes de la enseñanza media $-1^{\circ}$ y $2^{\circ}$ de BUP y FP de primer grado-.

Sin embargo, la LOGSE anunciaba cambios sustantivos que no han terminado de concretarse. El primer aspecto que quiero señalar es el del profesorado. A pesar de todo lo proclamado, la LOGSE y la reforma en general no han incrementado los niveles de autonomía profesional de los grupos de docentes. Esta autonomía debe concretarse fundamental pero no exclusivamente en los proyectos curriculares de centro. Estos documentos debieran ser la expresión del modo en que los claustros y/o los departamentos -o profesores de un ciclo- interpretan el Diseño Curricular Base. Esta autonomía curricular relativa es consecuencia de la necesidad de que cada centro analice el tipo de alumnos que escolariza -cuál sea su lenguaje, su cultura, su familia, su cosmovisión, etc.- para a partir del conjunto de peculiaridades del alumnado -cuyo conocimiento requiere la colaboración de toda la comunidad educativa: profesores, alumnos y sus padres- alcanzar los objetivos que para cada nivel educativo marca la ley. Incluso los propios objetivos de la ley deben ser concretados en los proyectos curriculares en consonancia con el proyecto educativo de centro -documento este último fruto del consenso de todos los sectores de la comunidad educativa-. Por ello, en su momento, se aventuró que los libros de texto y demás material homogeneizador -o, si se prefiere, de hegemonía cultural de la clase dominante- dejarían de ser los amos y señores de la actividad escolar. En su lugar se fomentaría la lectura autónoma por parte de los alumnos, la búsqueda y uso de bibliografías alternativas, la potenciación de las bibliotecas de centro y de aula, etc. Nada de esto es una realidad y ahí tenemos los datos que prueban el escaso interés lector de la sociedad española y de los españolitos en particular.

No obstante, esta lamentable realidad, observadores miopes, como la Academia de la Historia, en su tétrico informe sobre la enseñanza de la historia en España considera que con la reforma educativa se prima el cómo enseñar en detrimento del qué enseñar. Por desgracia, lo cierto es que en el día a día de nuestra escuela prepondera una pedagogía de corte transmisivo (Freire la llamaba bancaria) en la que el profe- 
sor y/o el libro de texto monopoliza el espacio del aula. Los académicos manifiestan no saber algo tan elemental como es lo que pueda ocurrir dentro de las aulas. Si bien es cierto que apenas disponemos de investigaciones de corte etnográfico (cada vez más difíciles de realizar debido a la oposición creciente del profesorado a que un extraño, por muy investigador educativo que sea, entre en sus aulas) disponemos de algunas. Bastaría con haber consultado el interesantísimo libro de Raimundo Cuesta Fernández, Clío en las aulas. La enseñanza de la historia en España entre reformas, ilusiones y rutinas ${ }^{1}$ para comprobar la cera que arde en nuestros institutos de secundaria. Aquí se cita, entre otras investigaciones, la tesis doctoral de Nieves Blanco García del año 1992 en la que se dice que "habitualmente la secuencia de desarrollo [de las clases] que se sigue es: lectura de párrafos del libro de texto-explicación-continuación de la lectura-nueva explicación. Manuel [el profesor] pide a algún alumno (con más frecuencia varones) que lea un determinado párrafo y va deteniendo la lectura para introducir su explicación; finalizada ésta, pide al mismo alumno o a otro, que continúe y de nuevo se repite el mismo esquema. Son las explicaciones a partir de la lectura del libro de texto las que dan origen a los apuntes. No es infrecuente que Manuel indique a los alumnos que copien lo que va a dictarles, aunque hay también códigos no verbales que los alumnos interpretan para decidir lo que han de anotar y lo que no: cambios de tono y ritmo de voz".

En definitiva, poco se ha hecho por alterar las relaciones sociales dentro del aula. La vida en nuestras aulas -sobre todo en la secundaria- sigue dominada por la pedagogía de la tiza y la pizarra, el tedioso e inútil dictado de apuntes, el subrayado -a instancias del profesor- en distintos colorines del libro de texto.

En este modelo es difícil que se pueda poner en práctica una de las claves de la reforma: la atención a la diversidad. Por desgracia, ya desde la época del gobierno del PSOE, la atención a la diversidad se ha entendido como una estrategia para librarse de los alumnos menos académicos por diferentes vías. Por ejemplo, la diversificación curricular -término capcioso allí donde los haya- es un conjunto de cursos de áreas instrumentales para mayores de dieciséis años. La opcionalidad curricular -y eso se vio muy claro en Cataluña- es un mecanismo para que "libremente" los considerados malos estudiantes se ahorquen con la soga de la pedagogía de perder el tiempo. Con este esquema interpretativo se obvia que todos los alumnos son diversos, que incluso los alumnos de alto rendimiento académicos son diversos; que, en definitiva, las adaptaciones curriculares deben afectar a todos los alumnos. ${ }^{2}$

En buena medida las anquilosadas prácticas pedagógicas citadas más arriba son el resultado de la nefasta formación inicial tanto del magisterio como de los profesores de secundaria. A diferencia de lo que ocurriera con la LGE de 1970, la LOGSE solo se refiere a la educación pre-universitaria. Dado que la reforma educativa en la universidad nada tiene que ver con el cambio en el estilo docente, nuestros futuros profesores siguen imitando las prácticas pedagógicas de los niveles educativos de mayor prestigio. ¿Cómo puede innovar en el aula quien ha pasado la mayor parte de sus estudios universitarios copiando apuntes? ¿cómo puede un maestro promover un ambiente de investigación en su aula si en su formación inicial no ha investigado -seguramente al igual que la mayoría de los profesores de su Escuela Universitaria de

1. Madrid, Akal, 1998.

2. Esto se puede ver en el capítulo 3 del libro de Mariano Fernández Enguita, Reforma educativa, desigualdad social e inercia institucional, Barcelona, Laia, 1987. 
Magisterio-? Sobre los profesores de secundaria es de sobra sabido que carecen de sólidos conocimientos de pedagogía -salvo que por tal consideremos el Certificado de Capacitación pedagógica, el cual algo va mejorando-.

La formación permanente adolece de serios problemas. A mi juicio el principal es que las autoridades educativas han promovido el cursillismo orientado hacia una absurda cruzada de alfabetización en la reforma, cuyos sacerdotes han sido los CPRs y toda una caterva de predicadores atiborrados de psicologismo y pedagogismo desmesuradamente abstractos cuando no absurdos (a todo el mundo le sonarán expresiones tan ingeniosamente chocarreras como panel vertical de conocimientos o segmento de ocio). Para colmo de males, desde la Administración del PSOE no se dudó en culpar a los profesores del posible fracaso de la reforma. Jaume Martínez Bonafé ${ }^{3}$ señalaba que los gestores de aquella Administración han difundido la idea de que lo que pasa en las escuelas, sobre todo, lo que pasa de malo en las escuelas, es porque los profesores no saben, no han aprendido o no han querido aprender lo suficiente para hacer realidad aquella "realidad" social-educativa idealizada. Pone como ejemplo de esta actitud los contenidos aparecidos en el no 223 que la revista Cuadernos de Pedagogía dedicó monográficamente a la valoración institucional de los "proyectos curriculares de la Reforma", que firman C. Coll y E. Martín, entonces Director General de Renovación Pedagógica y Directora del Gabinete de la Secretaría de Estado de Educación. Martínez Bonafé invita al lector a juzgar las valoraciones que allí se hacen de las dificultades de implementación de esto que llamaros "segundo nivel de concreción del nuevo modelo curricular".

García Garrido ${ }^{4}$ señalaba, en el marco de una comparativa con el resto de Europa, que España es un país en el que es especialmente necesaria la reforma de la formación inicial del profesorado. Consideraba que el déficit de la formación permanente es todavía mayor. Aquí creo que es donde es más fácilmente detectable el escaso empeño que el PSOE ha puesto en cambiar los cimientos de la dominación social. Un profesorado -tanto el de primaria como el de secundaria- escasa o insuficientemente preparado garantiza la existencia de una escuela en la que solo se podrán salvar los ya elegidos previamente. Bourdieu decía que la escuela valora aquello que ella misma no está en condiciones de enseñar, lo que, con esta formación del profesorado, solo se puede aprender fuera de la escuela: maneras de hablar, de expresión corporal, etc., elementos estos con los que es posible avanzar sin grandes dificultades en la escolar carrera de obstáculos.

Como era previsible, en los últimos tiempos ha estallado la bomba de relojería de la comprensividad. Estamos muy lejos de haber resuelto el problema de la educación obligatoria, especialmente de los cuatro cursos de la ESO. Aún sigue vigente la idea y la práctica de que la ESO es un nivel propedeútico para el bachillerato en detrimento de sus funciones de preparación para la vida activa -y adulta- y para la formación profesional de grado medio. De hecho, la gran divisoria en nuestro sistema educativo no es la ESO frente al Bachillerato o el Bachillerato frente a la Universidad, sino la educación obligatoria frente a la educación post-obligatoria. Aquella, como su propio nombre indica, es la que ha de cursar, lo quiera o no, todo ciudadano. Aquí se encuentran las claves del tipo de persona qué queremos formar. Con este nivel educativo se deben adquirir una serie de destrezas -enumeradas de un modo muy impre-

3. ¿Enseñar a los maestros?", Kikirikí, 52.

4. Papeles de la economía española, 86, 2000. 
ciso en la ley- que permitan acceder a la vida activa -no necesariamente al desempleo o al sub-empleo- y sobre todo se debe haber aprendido a aprender. Es decir, como consecuencia de haber transitado esos diez años obligatorios por el sistema educativo nuestros jóvenes deberían ser capaces de saber buscar la información que precisan, saber expresarse con corrección por escrito y oralmente, saber desenvolverse con cierta fluidez en inglés, etc. Todos sabemos que nada de esto es cierto: en la mayor parte de los casos quien abandona el sistema educativo a los dieciséis años es un pésimo estudiante que difícilmente acudirá a un librería -ni siquiera, como diría Woody Allen, cuando llueve-.

\section{La opinión del profesorado ${ }^{5}$}

Al hilo de una jornadas de reflexión en diciembre de 1999 sobre - o, más bien, contra- la LOGSE, el ministerio de educación abrió un foro de debate virtual sobre la educación secundaria. Se trata de más de quinientas opiniones en su mayoría de profesores. En este foro preponderan las opiniones contrarias a la comprensividad, lo que podría ser indicativo de que hay mayoría de profesores de medias opuestos a la reforma -lo que a su vez exigiría cambios radicales de la ley- o que simplemente estamos asistiendo a eso que Noelle-Neumann ${ }^{6}$ llamaba la espiral de silencio (una situación en la que se oye fundamentalmente la opinión de quienes mantienen una postura, mientras que el resto, la mayoría, permanece en un prudente silencio). Teniendo en cuenta que este foro se inserta en la página web de la sectaria reunión auspiciada por el MECD y que la reforma no parece suscitar entusiasmos desmedidos -a diferencia de lo que ocurre con las opiniones adversas- es bastante probable que estemos en presencia del fenómeno de la espiral del silencio.

Apenas sabemos nada sobre las personas que intervienen en el debate. Sabemos que son profesores de secundaria en su mayoría -o un genérico otros para los que no lo son-, su sexo -por el nombre de pila- y algún dato que suministran en su texto (soy catedrático, agregado, de Izquierda Unida, etc.). Sin embargo, no sabemos en qué tipo de centros trabajan, cuál es su experiencia docente, etc. En definitiva, ya que para participar en el foro se exige identificarse, no hubiera estado mal completar algunas cuestiones previas que hubiesen permitido situar y clasificar a los participantes. En un debate de estas características haber sido anteriormente profesor de BUP-COU o de FP es un dato clave.

En las opiniones vertidas por el foro son varias las referencias a los mecanismos de selección de los participantes: desde el desconocimiento total hasta las acusaciones directas al sectarismo de las autoridades educativas La intención, para muchos de los participantes, es clara: degradar la LOGSE. Los partidarios de la LOGSE opinan que desde que gobierna la derecha esta ley está siendo torpedeada.

Los detractores de la LOGSE han encontrado en este debate la posibilidad de desfogarse, recurriendo en multitud de ocasiones a la descalificación del adversario. Según ellos, los defensores de la comprensividad están fuera de las aulas: predican, pero no dan trigo.

5. Se puede leer una versión mucha más amplia del contenido de este epígrafe en el primer número de 2001 de la Revista de educación.

6. E. Noelle-Neumann, The Spiral of Silence. Public Opinion: Our Social Skin, Chicago, University of Chicago Press, 1984 (Hay versión española en Paidós). 


\section{A) Una sociedad hedonista}

Una queja frecuente en este debate ha sido la de considerar que la sociedad, y muy en particular los padres, desprecia la labor de los profesores y por extensión la cultura académica. Los docentes se consideran profesionales desprestigiados y mal pagados -coas, esta última, sobre la que habría que introducir muchas matizaciones- La expansión de la educación secundaria inferior a toda la población siempre implica caer en el riesgo de la devaluación de este título escolar, tanto en el mercado de trabajo como en de prestigio: más cantidad se hace equivaler a menos calidad. El efecto perverso de esta extensión es que parte de los grupos sociales que anteriormente no frecuentaban la secundaria perciben la escasa utilidad instrumental de la permanencia de sus hijos en un sistema educativo que con más frecuencia de la deseada le lanza mensajes recriminatorios. A ello hay que añadir que la dimensión expresiva de la escuela -la relevancia y aplicabilidad de los conocimientos y destrezas escolares- es más bien nula. Por otro lado, los grupos sociales que desde hace varias generaciones tienen garantizada la secundaria abandonan la enseñanza pública -cuya calidad apenas ha mejorado a pesar de los buenos propósitos de la reforma- dirigiéndose hacia centros privados o, por lo menos, concertados. ${ }^{7}$

Hace unos cuantos años, el sociólogo conservador norteamericano Daniel Bell explicó en su libro Las contradicciones culturales del capitalismo que las sociedades capitalistas vivían la contradicción que suponía por un lado el espíritu frugal, de ahorro y de sacrificio necesarios para la inversión capitalista y por otro lado el desarrollo desde comienzos de siglo de una sociedad de consumo en la que se incentiva el hedonismo, elemento sin el cual no se consumirían los frutos de la frugalidad. Bell señalaba que el principio axial del la cultura moderna es el desarrollo del "yo". Este tipo de contradicciones aparece en algunas de las intervenciones del foro.

Los padres son uno de los blancos predilectos de esta crítica a la indiferencia de la sociedad. Sin embargo, en ningún momento se explica qué podría hacer la escuela para combatir esa indiferencia.

\section{B) Segregación/integración}

Entre los críticos de la ESO hay unanimidad en considerar un tremendo error hacer compartir las mismas aulas a alumnos con muy diferentes rendimientos académicos. De acuerdo con esta postura simplemente sucede que aproximadamente a los catorce años de edad -para algunos quizás antes- los alumnos manifiestan distintos intereses y parte de ellos no desea aprender lo que la ley establece para los niveles obligatorios. Dado que no quieren estudiar la alternativa es separarlos del resto de los estudiantes -los que sí quieren estudiar- e introducirlos en programas de formación profesional -o pre-formación profesional-. Se trata de un tipo de explicación que se remite en exclusiva a la manifiesta falta de voluntad de seguir estudiando y el consiguiente boicoteo del normal desarrollo de las clases por parte de algunos estudiantes.

El aspecto más polémico de la reforma es el haber prolongado la comprensividad hasta los dieciséis años. En el modelo de la Ley General de Educación de 1970 a los catorce años se producía una escisión entre los alumnos de rendimiento académico admisible -los que obtenían el graduado escolar y podían pasar al BUP-y los de bajo

7. De hecho uno de los participantes en el debate virtual recomienda a los padres más preocupados que se lleven a sus hijos a la privada. 
rendimiento -quienes obtenían el certificado escolar y no podían acceder al BUP-. Ahora la situación es distinta y los antiguos profesores de bachillerato se ven obligados a bregar también con los alumnos menos académicos. Todo ello en un contexto en el que el primer nivel de educación secundaria, a diferencia de lo que ocurría hasta ahora, es obligatorio, lo que dulcifica la función selectiva de la escuela.

Son pocas las explicaciones que dan los detractores de la ESO al fenómeno del rechazo escolar, fenómeno del que por desgracia se quejan también los profesores de primer ciclo de primaria. Mayoritariamente las explicaciones se remiten, en el más craso de los psicologismos, a la mera voluntad de los alumnos. De este modo, simplemente sucede que unos quieren estudiar y otros no. La escuela oferta una cultura -para los defensores de esta posición, la cultura sin más- de la que unos desean disfrutar y otros no. No parece detectarse ningún condicionante como la clase social, el nivel cultural de los padres o similar que pudiera dificultar el acceso a la cultura escolar y mucho menos si se debería poner en duda el supuesto carácter neutral de la cultura académica.

A partir de aquí lo que se pide es la segregación de los alumnos de menor rendimiento. Por desgracia, la propuesta no va más allá de que se los quiten de encima. Desaparece la idea de que, al menos en los niveles de educación obligatoria, todos los alumnos son educables. Aunque no se plantea en estos términos, la conclusión lógica de estas propuestas es que no todos los ciudadanos están en condiciones de alcanzar los objetivos que marca la ley para la educación obligatoria.

El que en la reunión sobre la ESO se haya invitado a un ponente alemán para hablar sobre el sistema educativo de su país ha dado alas a los partidarios de la segregación temprana. El sistema alemán -al igual que el austriaco- es una excepción en el ámbito europeo. Los expertos educativos -y la propia OCDE, organización que nadie en su sano juicio podría tildar de progresista- coinciden en señalar los efectos nefastos de una especialización escolar temprana. Lo que los empleadores demandan a los egresados de la educación secundaria es sobre todos capacidad de adaptación para un mundo productivo en continuo cambio y no personas especializadas en ámbitos que previsiblemente dejen de existir como tales al cabo de pocos años -incluso antes de que los estudiantes salgan de la escuela-.

Esta segregación provoca protestas por parte de los profesores de tecnología. No obstante, y como cabía esperar, los defensores de la LOGSE señalan los aspectos positivos de la mezcla de alumnos.

\section{C) Descenso del nivel y promoción automática}

A pesar de los que los datos -especialmente me refiero a los del INCE- ${ }^{8}$ de que se dispone muestran lo contrario, las quejas sobre el descenso de nivel que la ESO supone son moneda común entres sus detractores. El error consiste en comparar el nivel del anterior BUP- $1^{\circ}$ y $2^{\circ}$ de BUP- con la actual ESO - $3^{\circ}$ y $4^{\circ}-$. Esta compara-

8. Me parece que apenas se ha meditado sobre la terrible contradicción que supone impulsar una reforma basada en el constructivismo y valorarla con preguntas de tipo test. En el volumen titulado Diagnóstico general del sistema educativo. Avance de resultados publicado en 1998 por el INCE el método utilizado para determinar el grado de conocimiento de los alumnos se basa en preguntas con respuestas múltiples, muchas de las cuales rezuman ambigüedad o permitirían múltiples respuestas. Sin embargo, solo es posible una única respuesta correcta y, por supuesto, no cabe posibilidad alguna de diálogo con las cuestiones planteadas. 
tiva significa ignorar que en el sistema educativo de la LGE había alumnos del grupo de edad 14-16 años en BUP, en FP-I y, no se olvide, desescolarizados (en cualquier caso la comparativa no incluiría a estos últimos). No obstante hay quien con demasiada celeridad concluye la existencia de "varias promociones lastradas por el peso de la ignorancia".

El descenso del nivel educativo es el gran argumento de los detractores de la comprensividad. Es obvio que esta comprensividad va a plantear problemas. La enseñanza secundaria escolarizará a todos los estudiantes sin distingos de su posible perfil profesional o académico. Esto ha suscitado el debate sobre la egebeización de la secundaria, sobre el descenso del nivel educativo, etc. El lúcido estudio de Fernández Enguita ${ }^{9}$ sobre el debate en torno a la integración o la segregación aclara las cosas, al tiempo que rebate los diferentes trabajos que pretenden mostrar que este nivel ha descendido. Según la Fundación Carnegie, el nivel de aptitud verbal y matemática de los estudiantes norteamericanos de secundaria no ha dejado de descender entre 1952 y 1982. El problema de este estudio y de otros similares es que no tienen en cuenta que la tasa de asistencia a la enseñanza secundaria ha aumentado, lo que supone la diversificación del estudiantado y el consiguiente incremento del número de ndo crecientemente alumnos menos motivados académicamente, y por tanto, necesariamente, un descenso de la media.

La promoción automática -el hecho de que no se puede repetir más de dos cursos a lo largo de la educación obligatoria- se ha convertido en una de las bestias negras para parte del profesorado. En general las opiniones contrarias a esta promoción supuestamente automática consideran que el principal mecanismo de incitación al trabajo escolar es la amenaza de la evaluación, lo que trasluce bien a las claras que algunos consideran que el trabajo escolar es algo que en sí mismo no puede interesar a nadie, lo que obliga a sacar a relucir el discurso de la amenaza, de la disciplina, de las notas, etc.

Un problema no resuelto es el de asignaturización del conocimiento. No es infrecuente que un profesor de Lengua considere que un estudiante tiene escasas competencias lingüísticas mientras que el profesor de Ciencias Sociales tiene una opinión opuesta. A ello hay que añadir el excesivo número de asignaturas. En este contexto de exceso de asignaturas son varias las propuestas de reducir su número empezando por la religión.

\section{D) La superación de la vieja pedagogía}

La apuesta por la comprensividad es al mismo tiempo una apuesta por una nueva pedagogía. Hablar en estos términos es despertar la desconfianza de muchos profesores que manifiestan haberse tropezado con ideas pedagógicas inviables.

Las innovaciones pedagógicas sugeridas por la LOGSE no se han convertido en realidad, a pesar de sus enormes potencialidades. Una de la claves de la LOGSE consiste en la superación de la pedagogía basada fundamental o exclusivamente en la mera transmisión de conocimientos desde el profesor -o el libro de texto- a los alumnos. Lo que ahora se demanda es una educación que promueva el trabajo autónomo de los estudiantes -obviamente bajo la guía del profesor- en el entendido de que la mejor manera de adquirir conocimientos significativos consiste en

9. Integrar o segregar. La enseñanza secundaria en los países industrializados, Barcelona, Laia, 1986. 
alentar la experimentación, el espíritu de aventura. No otra cosa es el espíritu científico que la escuela debe alentar. La experiencia escolar debe conducir a la pasión por el conocimiento, de modo que el aprendizaje es una actividad que continuará durante toda la vida de los ciudadanos. De hecho en el debate varias opiniones reflejaban la idea de que un profesor que meramente transmita conocimientos está condenado a ser sustituido por máquinas.

\section{E) Conclusiones de este debate}

Uno de los elementos más graves que cabe concluir de este debate es la existencia de un elevado número de profesores que no cree en el espíritu de la LOGSE. Mientras que esto sea así la reforma no podrá llevarse a cabo. Al fin y al cabo la reforma insiste en la autonomía del trabajo en grupo de los docentes, trabajo que se concreta entre otros documentos en el proyecto curricular. Si no es posible distinguir, como ocurre hasta ahora, el proyecto de un centro del de otro; si las editoriales de libros de texto siguen haciendo su agosto al calor de la reforma; si el uso de las bibliotecas de aula -cuando las hay- o de centro es más bien escaso; si los centros siguen siendo búnkers cerrados a su entorno, etc, es que algo muy grave está ocurriendo en nuestro sistema educativo. Por desgracia los temas relacionados con la educación no gozan de prioridad en la agenda política -a pesar de la insistencia en la educación de nuestro presidente regional-. Si fuera así, los contenidos de este debate tendrían que ocupar a los medios bastante más que la información deportiva.

En las intervenciones contrarias a la comprensividad parece detectarse una defensa numantina del carácter elitista de la educación secundaria: no todos pueden saborear las mieles de la cultura académica. Por desgracia, estas críticas tienen mucho de verdad. Sin embargo no se deben plantear desde la secundaria obligatoria. Si un estudiante llega a la ESO sin saber leer ni escribir a quien habría que pedir cuentas es a la educación primaria ¿Cómo es posible que Ilegue un analfabeto a la ESO?, ¿si hay niños que después de ocho años en la escuela no saben ni leer ni escribir es que nos hemos equivocado al considerar que todos deben pasar por la escuela? Si no es así, ¿por qué no se han disparado todas las alarmas?, ¿por qué los profesores no se movilizan frente a esta situación? El problema no está en la promoción automática. Lo está en la inflexibilidad de la escuela, en su incapacidad para adaptarse a los diferentes entornos. ¿Dónde quedan las adaptaciones curriculares? Decía Claude Grignon, en una entrevista reciente, que una "verdadera reforma sería aquella que de forma realista tuviera en cuenta las grandes distancias que existen entre la cultura de los recién llegados a la escuela y la cultura que esta continúa transmitiendo". ${ }^{10}$

Muy grave es la queja generalizada de que la reforma se han planteado desde los despachos. Incluso desde quienes defienden la LOGSE esto se percibe así.

(...) después de una fase de espontaneísmo militante en los años 70, en la década siguiente, la de la "feliz gobernación" socialista, vino a darse una cierta oficialización de los efectivos renovadores, que, en buena parte, se vieron seducidos por un lenguaje familiar (auténticos cantos de sirena de la vanguardia pedagógica) ahora bendecido por los halagos y recompensas del poder. De esta suerte, muchos docentes vinculados a los movimientos de renovación pedagó-

10. Archipiélago,38, 1999, pp. 13-15. 
gica pasaron a engrosar las multiplicadas filas del ejército ministerial de cuadros técnicos encargados de materializar los afanes reformistas, lo que, como es sabido, originó una más que babélica confusión entre saberes y poderes."

(...) los psicólogos introdujeron una verdadera inflación de lenguajes que desviaron la atención de los problemas importantes. Creer que por el simple hecho de formular conceptos y teorías psicológicas se derivan cambios en la realidad es una alucinación muy común en los "expertos" y fue muy fácil políticamente. La jerga terminológica sirvió para rellenar vacíos y supuso un placebo importante. En lugar de acometer reformas de gran calado, como era la de la formación del profesorado, la carrera docente, la dotación de centros, la dotación de bibliotecas, el combate contra las desigualdades, etc. se gastaron decenas de miles de millones en hacer reuniones, en llenar las administraciones de "liberados" dedicados a hacer guiones que traducían los lenguajes al uso para enviar a los profesores, unos lenguajes que, por otro lado, iban cambiando como si con ello hubiese que garantizar la pervivencia de los que vivían de eso más de la continuidad de la política. ${ }^{12}$

En definitiva, estamos ante una situación compleja. Tropezamos con la dificultad más esperada de la LOGSE: la educación secundaria. Multitud de fallos han posibilitado el estallido de descontento de los antiguos profesores de bachillerato que no terminan de ver nada claro por qué se derogó la LGE de 1970.

\section{Objetivo prioritario del gobierno: la contra-reforma de la LOGSE}

El partido en el gobierno ha tratado de buscar cobertura en su intento de acometer la contra-reforma. Quizás el escenario más destacado de este intento fueron las jornadas de reflexión sobre la LOGSE a las que nos referimos en el apartado anterior.

Merece la pena destacar la intervención del entonces director del Departamento de Análisis y Estudios del Gabinete de la Presidencia del Gobierno, Eugenio Nasarre y las conclusiones de las jornadas ponen claramente de manifiesto la voluntad del gobierno.

A este gobierno le resulta intolerable que la educación, desde la secundaria, no sea un elemento de selección social. En este respecto la primera gran batalla es la librada en favor de la escuela concertada y privada. Su claro propósito es reservar los centros públicos para los alumnos en situación de riesgo, cuyos padres tengan menor nivel educativo y económico, minorías étnicas, etc. El famoso punto que las entidades titulares de los centros concertados pueden asignar a las solicitudes de matriculación ha intensificado la capacidad de selección del tipo de alumnos con que desean contar. Una enseñanza pública que pierde diversidad social -y, en consecuencia cultural- es una enseñanza abocada al fracaso. Si bien es cierto que esta segregación también condena a los centros concertados a una escasa diversidad les permite quedarse con los mejores alumnos, lo que favorece unos mejores resultados académicos. Esto les

11. R. Cuesta y J. Mainer, "Encrucijadas y paradojas de los cambios educativos", Archi-piélago, 38, 1999, p. 24.

12. Entrevista a José Gimeno Sacristán en Archipiélago, 38, 1999, p. 32. 
permite incidir en los conocimientos -en definitiva en el mensaje del eficiencismo escolar- en detrimento de la solidaridad, la cohesión social o la eficacia del conjunto del sistema educativo.

Una vez dado este primer paso, ahora el Gobierno pretende afrontar una reforma de la LOGSE que rompa su carácter comprehensivo para los estudiantes de entre 14 y 16 años de edad o, lo que es lo mismo, el segundo ciclo de la ESO -lo que en términos de grupo de edad equivalía a $1^{\circ}$ y $2^{\circ}$ de BUP-.

En su ponencia, Eugenio Nasarre hace una digresión histórica sobre el significado de la educación secundaria. La ESO no puede ser una mera prolongación de la primaria. La educación secundaria obligatoria, concluye precipitadamente, no puede ser igual para todos:

Pensar que, en aras de la igualdad de oportunidades, todos deben de seguir las mismas enseñanzas -con la única variación de las materias optativas- hasta los 16 años y que sólo a partir de esa edad se pueden establecer diversificaciones del currículo para los alumnos que lo necesiten, constituye una concepción equivocada de la igualdad de oportunidades.

La ministra, en su intervención en el club Siglo XXI, hablaba, con una temeraria falta de precisión lingüística, de un "igualitarismo mal entendido ante el aprendizaje".

Señalaba Nasarre que hay que precaverse contra un "modelo de comprensividad de carácter radical" -se supone que el tenemos actualmente es radical- y empezar a pensar en una "diversificación inteligente" -la actual quizás sea estúpida-. Para ello nada mejor que remitirse a los supuestos deseos de los alumnos, los cuales son invitados a manifestar sus "intereses, vocaciones y aptitudes". De nuevo estamos ante el discurso esencialista/psicologista según el cual la escuela, de un modo neutral, descubre que hay alumnos "objetores" a la enseñanza de régimen general y a los que hay que recluir en algún lugar donde no contaminen a los buenos estudiantes. Ese lugar es la diversificación curricular. En definitiva, se trata de que no estudien lo mismo que los demás ni con los demás. Nasarre se pregunta retóricamente: "¿Es preciso que sean los mismos objetivos para todos los alumnos?". De la respuesta a esta pregunta se deduce si consideramos o no que todos los niños son educables, si creemos que de la educación obligatoria deben salir ciudadanos dotados de un espíritu inquisitivo, si todos pueden aprender a aprender.

Segregar en los niveles obligatorios de la enseñanza es condenar al fracaso a los no elegidos. Lo que propone Nasarre es olvidar la necesidad de la flexibilidad curricular para todos los alumnos, no solo para los de menor rendimiento. No se trata de que los niños se adapten a la escuela, sino de que la escuela se adapte a ellos. La diversificación curricular, la educación compensatoria, la garantía social o demás vías segregadoras solo sirven para negar la diversidad de culturas que existe en nuestra sociedad.

Más bien deberíamos preguntarnos por qué no funcionan los proyectos educativos, por qué los proyectos curriculares rara vez reflejan las peculiaridades del tipo de alumnos de cada centro, por qué las editoriales de libro de texto siguen siendo un negocio altamente lucrativo. Esta preguntas no se las puede hacer un gobierno de derechas cuyo principal interés es proyectar una ideología meritocrática, individualista, en la que cada cual es responsable de su trayectoria escolar.

¿Se habrá preguntado el señor Nasarre alguna vez por qué el fracaso escolar se concentra en ciertas clases sociales y no en otras? Y si lo ha hecho, ¿qué respuesta -o respuestas- ha encontrado? 
A la ponencia de Nasarre hay que añadir el escalofriante documento de conclusiones de las jornadas. En él nos enteramos que habría que introducir en el currículo una Historia de las Religiones. Puestos a buscar carencias en el currículo, ipor qué no mejor una introducción al Derecho o a los medios de comunicación? Y si pensamos en materias que ya se abordan en Ciencias Sociales, ¿por qué no una historia del Estado o de la tecnología?

Mucho más grave me parecen las conclusiones obtenidas "de conformidad con los análisis del profesorado, basados en los resultados de la experiencia". De las catorce conclusiones nada más y nada menos que siete se refieren a la disciplina: el alumno debe conocer la importancia de la disciplina, el rechazo a la institución escolar genera comportamientos agresivos o problemas de disciplina, aumento de la indisciplina en muchos centros, existencia de conflictos, la normativa de convivencia debería ser más breve -recortada deberían decir- y clara, refuerzo de la autoridad del profesorado, hay que buscar soluciones a los "objetores escolares". Si realmente este fuera el sentir mayoritario del profesorado deberíamos pensar seriamente en la desescolarización (por cierto injustamente vapuleada en algún artículo de esta revista). Una escuela cuyos conocimientos no resultan atractivos para la mayoría de los estudiantes no tiene más remedio que recurrir a una disciplina punitiva.

Aunque ni en las conclusiones ni en el texto de Nasarre se abordó, otra cuestión que afecta a la LOGSE es la posibilidad de que se establezca una especie de reválida al finalizar la ESO. Personalmente no tengo nada que objetar a que al finalizar la ESO -o una carrera universitaria- haya que pasar una prueba de madurez. Este sistema -que nada tiene que ver con las intenciones del gobierno- es el que se pone en práctica en la experiencia innovadora de la escuela de secundaria Central Park East. Aquí los exámenes convencionales ceden su lugar a lo que se denomina Graduación por historiales de evaluación del alumnado, historiales que son evaluados por un Comité similar a los de nuestras tesis doctorales. Se trata de un comité de cinco personas, tres de ellas profesores del colegio, un estudiante y un adulto elegido por el estudiante (adulto que puede ser quienquiera que determine el alumno: su padre, su madre, un conocido, un líder de la comunidad, otro profesor, etc.). La tarea de este comité es leer, revisar, escuchar los datos contenidos en los catorce historiales (más o menos correspondientes a áreas curriculares) que presentan los alumnos. Estos se ven obligados a defender oralmente y en público sus planteamientos. En definitiva se trata de una forma de trabajo que está en contra de la simple memorización de la materia.

Sin embargo, lo que propone el gobierno es otra cosa muy distinta. Una reválida al finalizar la ESO condiciona toda la pedagogía, todos los contenidos, actitudes y procedimientos de la secundaria obligatoria. Sería el final definitivo de cualquier discurso sobre la atención a la diversidad o la flexibilidad curricular.

A todo esto faltaría por añadir la polémica suscitada por la pretensión del paso directo desde los ciclos formativos de grado medio a los de grado superior. Actualmente acceden a los ciclos de grado superior los bachilleres o desde el mercado de trabajo -en este último caso realizando una prueba específica-. Proponer la continuidad directa del grado medio al superior es desprestigiar -y seguramente vaciar de contenido- a una buena vía de especialización para quienes hayan cursado y aprobado el bachillerato. 
Fruto de este tipo de reflexiones y en espera de lo que pueda dar de sí la futura ley de calidad de la enseñanza el ministerio acaba de sacar adelante unos decretos de mínimos curriculares para toda la enseñanza media -la ESO y el bachillerato-. Se trata de un decreto que vendría a sustituir al DCB. Entiendo que lo aquí planteo es aproximadamente generalizable a las demás áreas y asignaturas. Las propuestas del gobierno pretenden hacer más rígidos, menos susceptibles de interpretación, los componentes cognitivos del mensaje escolar. Hasta ahora, al menos en teoría, tenemos un modelo curricular flexible, de modo que las autoridades educativas -el Ministerio y las Comunidades Autónomas- elaboran el denominado Diseño Curricular Base (DCB), el cual debe ser concretado y desarrollado por los profesores -departamentos o maestros de ciclo-, de modo que estos sean capaces de adaptar el currículo explícito a las peculiaridades del tipo de alumnos escolarizados en cada uno de los centros educativos. A ello habría que añadir la tercera fase de concreción curricular: la programación de aula. Eso explica que el DCB en realidad no consista, a diferencia de lo que ahora se propone, en una secuenciación de contenidos por curso. El modelo hasta ahora existente confiaba en la capacidad de trabajo autónomo de los profesores -considerados individual y grupalmente-, a pesar de que, en la mayoría de los casos, el currículo ha sido elaborado por las editoriales de libros de texto.

Me referiré en concreto al área de "Ciencias Sociales, Geografía e Historia" en la Educación Secundaria Obligatoria. Si se lee la introducción - la presentación del área- lo primero que sorprende es por qué denominar también "Ciencias Sociales" a esta área. En realidad solo se habla de Historia y de Geografía (por cierto, no se sabe muy bien por qué ora los autores -o autor- del texto que comentamos lo escriben con mayúscula, ora con minúscula). Además de ello Geografía e Historia son dos asignaturas totalmente distintas. Basta con ver el temario. En el primer ciclo de la ESO más o menos la mitad de cada curso se consagra a cada una de las disciplinas. Es tan sumamente visible esta separación que la cronología histórica se interrumpe bruscamente de modo que el final de un curso coincide con el comienzo del siguiente.

En $3^{\circ}$ de la ESO solo se estudia lo que se presenta como Geografía y en $4^{\circ}$ solo Historia. En tercero el programa entra de lleno, con sendos temas, en cuestiones de economía, sociología y política. Sin embargo, en ningún momento se explica el papel de estas ciencias en la configuración de la asignatura "Ciencias Sociales, Geografía e Historia". No parece claro que en el tema 3 de $3^{\circ}$ de ESO se incluya el apartado "La organización territorial del Estado en la Constitución de 1978" y más abajo, en el mismo tema, se incluya "La diversidad geográfica de España". La distribución por autonomías no tiene porqué coincidir con la geográfica. No debemos confundir una división administrativa con otra geográfica.

En $4^{\circ}$ curso hay más elementos controvertidos. Así, el tema 7 -referido a la España del siglo XIX- incluye un epígrafe titulado "Arte y Cultura del siglo XIX". Debería añadir "en España", ya que en el siguiente tema hay un epígrafe titulado "La cultura europea del siglo XIX". Incomprensiblemente ha desaparecido el arte. Resulta difícilmente explicable que se hable de "arte y cultura en el siglo XX" -esta vez ha reaparecido el arte- en el tema dedicado al periodo de entreguerras -palabra esta última que no se escribe con mayúscula-, periodo que como su propio nombre indica solo abarca unos pocos años -veinte- del breve siglo XX -breve, pero no tanto-. 
Siguiendo en 4º curso podemos observar que, aunque se habla de la "Segunda Guerra Mundial", no hay ningún epígrafe dedicado a esta conflagración. En el tema 12 ("El mundo occidental") no estaría de más incluir a Japón. El tema 14, dedicado al franquismo, propone el análisis del régimen desde el prisma de la relaciones internacionales y no su estudio en sí con carácter sustantivo (El régimen político y las relaciones internacionales"). Al estudiante se le invita a "subrayar la trascendencia de la construcción europea", a "caracterizar los profundos cambios y los cambios posteriores a la II guerra mundial" -ahora con minúsculas- o a "comprender y valorar los principios e instituciones básicas de los sistemas democráticos y reconocerlos en la vigente Constitución española". Sin embargo, no hay ningún criterio de evaluación -de los doce enumerados- que haga referencia al franquismo.

En esta asignatura ya tropezamos de lleno con los tres itinerarios educativos bachiller, formación profesional y abandono- que en breve el PP propondrá para acabar con el carácter comprehensivo de la ESO. Así, se dice que el estudio de esta asignatura ha de establecer "una base suficiente que les permita [a los alumnos] profundizar posteriormente en los temas tratados en sus estudios de Bachillerato, o a través de otros cauces académicos o de carácter autodidáctico".

Viendo la relación de temas propuestos para cada uno de los cursos cabe preguntarse dónde queda la autonomía curricular o la adaptación del currículo al entorno. Lo que aquí se impone es un temario cerrado que presumiblemente rellenarán las editoriales de libros de texto, cuyo agosto -o más bien septiembre- queda garantizado.

Los criterios de evaluación se podrían resumir en "apréndase usted los temarios uno tras otro y no se le ocurra alterar su orden" o "apréndase la lección". Estos criterios son el correlato de cada tema, es decir, una macro-pregunta relativa al tema en cuestión en donde se trata de garantizarse que el alumno ha aprendido lo que tiene que aprender y que no saca los pies del tiesto. Desaparecen de un plumazo, y sin que haya una reflexión sobre ello, los procedimientos y actitudes, en favor de lo que este gobierno, y en esto coincide con la concertada, considera el núcleo duro de la enseñanza: los contenidos.

En esta línea cabe entender la propuesta que la ministra ha hecho ya en varios escenarios: la de convertir la educación infantil en obligatoria. En su obsesión por acabar con el supuesto descenso de nivel, la ministra propone que los niños hayan aprendido a leer a los seis años. Por esta vía, toda la capacidad de innovación que hasta ahora estaba demostrando la educación infantil se vendría abajo.

\section{Conclusiones. La educación en una época conservadora}

En una reciente entrevista, Michael Apple hablaba de la confluencia de diversas tendencias a las que otorgaba la etiqueta de "modernización conservadora". Este es un movimiento que trata de redefinir la educación. Fundamentalmente son dos los grupos que lideran esta alianza: los neoliberales y los neoconservadores.

Los "neo-liberales" son modernizadores económicos que desean que la política educativa dependa de la economía, de la consecución de objetivos basados en una conexión más estrecha entre la escuela y el trabajo retribuido. Consideran que las escuelas deben estar conectadas con el mercado, especialmente el mercado capitalista global, y las necesidades y procesos laborales de tal mercado. Las propias escuelas precisan ser transformadas y convertirse en más competitivas 
poniéndolas en escenarios mercantiles por medio de los cheques escolares, los créditos sobre impuestos y otras estrategias mercantiles similares. En el caso español este grupo estaría claramente representado por la patronal de la enseñanza privada ACADE, la cual, por medio de un informe elaborado por Ramón Tamames, plantea la introducción del cheque escolar.

El segundo grupo son los neo-conservadores. Los neo-conservadores suelen estar de acuerdo con los neo-liberales en la importancia de la economía, pero su principal preocupación es la "restauración" cultural. Desean el regreso a un conocimiento de alto estatus dominado por el profesor, basado fundamentalmente en las tradiciones que históricamente han sido contempladas como el conocimiento legítimo de las universidades de élite. Debido a esto los neo-conservadores están profundamente comprometidos en la pretensión de establecer mecanismos más estrictos de control del conocimiento, de la moral y de los valores recurriendo a un currículo estatal o federal o a exámenes (muy restrictivos) estatales. Esta propuesta se basa en una desconfianza muy fuerte en los profesores y en los administradores escolares locales. Piensan que solo a través del establecimiento de un fuerte control central conseguirán su lugar en el currículo el contenido y los valores del "conocimiento legítimo". Junto a ello existe un compromiso con un currículo supuestamente más riguroso, basado en lo que consideran "altos estándares". De este modo, la propia escuela debe ser más competitiva, de manera que los estudiantes sean re-estratificados a partir de lo que sería visto como un "conocimiento neutral" y exámenes "neutrales" de rendimiento. En esencia, esto se convierte en un retorno a los principios del social-darwinismo en la educación. En la mayoría de los países, el neo-conservadurismo se conecta con el neo-liberalismo al conceder a los neo-liberales los mecanismos para determinar lo que sea una "buena escuela" y una "mala escuela" de manera que el mercado pueda condenar a la desaparición a las "malas escuelas".

En este marco podemos comprender la obsesión del ministerio por elaborar un currículo estandarizado y por elaborar unas instrucciones precisas para la redacción de los libros de texto, los cuales serían definitivamente entronizados como los amos y señores de los contenidos curriculares.

El informe que emitió la Academia de la Historia a medidados de 2000 cae de Ileno en esta modernización neo-conservadora. El informe se queja de un mal generalizado: la ignorancia de los alumnos después de haber cursado historia muchos años. Esto mismo ocurre con el resto de las áreas curriculares y asignaturas. La ineficacia de nuestras escuelas, haya o no LOGSE de por medio, es tremenda. No se trata solo o fundamentalmente, como parece deducirse del informe, de los libros de texto o de los contenidos de la enseñanza de la historia, sino del funcionamiento de nuestras aulas.

En el informe no se analizan ni la educación primaria ni el primer ciclo de la ESO. Solo se preocupa de $3^{\circ}$ y $4^{\circ}$ de ESO y de los dos cursos de bachillerato, corriendo un inexplicable velo sobre lo que los alumnos aprenden con relación a la historia entre los 6 y los 12 años.

El informe manifiesta su preocupación por el hecho de que la enseñanza de la historia está al servicio de la conformación de la identidad nacional distorsionando, si es preciso hasta el dolor, los acontecimientos históricos. Esto ocurre no solo en las diversas comunidades autónomas -cuestión que es objeto de análisis en el informe- de España, sino que también ocurre en los contenidos curriculares de historia en diver- 
sos países: bastaría comparar cómo se enseña la llegada de Cristóbal Colón a América aquí o en México.

En el informe se critica la inclusión de la historia a la altura de la ESO en el área más amplia de Geografía, Historia y Ciencias Sociales. A pesar de que los autores del informe se quejan de la posible disolución de la Historia en este mare mágnum, en realidad no ocurre tal cosa. Aunque el Diseño Curricular Base de esta materia pretende configurar un área de Ciencias Sociales, en la práctica y en la teoría está hegemonizada por la Geografía e Historia, que es la disciplina que han cursado la mayoría de los profesores de medias del área de Ciencias Sociales. No se entiende muy bien esta entronización de la Geografía y la Historia cuando los objetivos del área son básicamente tres: preparar a las nuevas generaciones para la convivencia en una sociedad pluralista y democrática -propósito que parece más propio de las Ciencias Políticas-, otorgar una dimensión humana y social frente al tecnicismo imperante labor propia de las Ciencias Sociales en general- y formar a los alumnos en el manejo crítico de la información -tarea más propia de las Ciencias de la Información, de la Semiótica, etc-. Los objetivos del área parecen más bien relegar a las Ciencias Sociales a un papel secundario.

El informe no tiene empacho en citar experiencias, sin duda aisladas, como la de ciertos profesores que "proponían a los alumnos ejercicios sobre países inventados, con datos imaginados de evolución de la población y de la producción". No obstante, mucho peor es la falta de fundamentación empírica de afirmaciones tan graves como a los centros de secundaria de Euskadi "asisten alumnos formados en las ikastolas en las que la historia que se enseña es de contenido parcial y tendencioso, inspirado en ideas nacionalistas favorecedoras del racismo y de la exclusión de cuanto signifiquen lazos comunes". Según parece, este racismo se manifiesta en apuntes y materiales manuscritos que suministran los profesores, apuntes y materiales de los que no se aporta cita alguna. En cualquier caso no debe perderse de vista que el suministro de apuntes y materiales forma parte de la autonomía docente de los profesores, de la que gozan no solo los profesores universitarios, sino también los de los niveles previos a la universidad.

Resulta sorprendente que la Academia se preocupe tanto por los contenidos de los libros de texto y no se interrogue sobre el sentido de este tipo de libros. A pesar de que en muchas ocasiones los autores -o coautores- de estos son profesores universitarios, nunca se cita a ninguno, cosa que sí se hace al hablar de los mentores de la escuela de los Annales o al referirse a los autores de libros no de texto - ¿o debería decir simplemente libros o libros científicos?-. A mi modo de ver uno de los serios problemas de los libros de texto es que rara vez incitan a ser trascendidos, a buscar información más allá de sus propios contenidos. Habitualmente presentan los hechos históricos y sus interpretaciones al margen de las controversias científicas. Esto es lo que explica que en ocasiones el autor o autores no aparezcan ni siquiera en la portada del libro. A diferencia de los que ocurre con su precio o su posible gratuidad, los contenidos de los libros de texto, salva raras ocasiones -como esta que estamos comentando- no son objeto de análisis por parte de la comunidad educativa. Se trata de libros que solo se pueden comprar en uno de los doce meses del año y, además, es prácticamente imposible encontrarlos en las bibliotecas. Es decir, son libros que carecen de interés para la comunidad científica -y para el ciudadano culto- y, sin embargo, son los libros con los que pretendemos formar a las futuras generaciones. 
Hasta ahora cuando se habla de alternativas al libro de texto se hace referencia a materiales curriculares aún más oscuros y particularistas como puedan ser los apuntes o las fotocopias. Sin embargo y sorprendentemente, es menos frecuente proponer el uso de la biblioteca de aula, la de centro, la de barrio o la especializada o simplemente el libro científico. Stenhouse hablaba de la "capacidad del estudiante para apelar a la biblioteca en contra del profesor". ¿Acaso no es posible que nuestros jóvenes puedan leer a Pierre Vilar o a Tuñón de Lara? ¿No sería preferible habituarles a los libros que cualquier ciudadano puede comprar en una librería en lugar de pastar en las páginas de los libros de texto? En definitiva, mi propuesta es alentar el trabajo autónomo de los estudiantes bajo la supervisión de sus profesores, los cuales de este modo verán reforzada su labor docente.

El futuro de los aspectos más innovadores de la LOGSE es muy incierto. El partido en el gobierno votó no a la LOGSE. Sin necesidad de cambiar la ley tiene el camino allanado para acabar con la comprensividad y la atención a la diversidad. Cuenta además con el apoyo de importantes sectores del profesorado que no han dudado en activar el discurso de la excelencia en cuanto han visto sus aulas invadidas por la chusma que antes se iba a la FP o a la calle. 